\section{High frequency of unruptured intracranial aneurysms in female patients with ischaemic heart disease}

Toshiyuki Uehara, Masayasu Tabuchi, Etsuro Mori
Neurology Service, Hyogo Brain and Heart Center at Himeji, Japan T Uehara

M Tabuchi

Department of Clinical Neurosciences, Hyogo Institute for Aging Brain and Cognitive Disorders, Himeji, Japan

E Mori

Correspondence to: Dr Toshiyuki Uehara, Neurology Service, Hyogo Brain and Heart Center at Himeji, 520 Saisho-ko, Himeji, 670, Japan.

Telephone 008179293

3131; fax 0081792927138 .

Received 26 February 1997 and in revised form 2 August 1997

Accepted 18 September 1997

\begin{abstract}
Objectives-Concurrence of coronary atherosclerotic lesions and intracranial aneurysms has been pointed out in several postmortem and clinical studies. However, the relative risk for intracranial aneurysms in patients with ischaemic heart disease remains uncertain. The aim of this study is to elucidate clinically whether ischaemic heart disease is a risk factor for intracranial aneurysms.

Methods-Between October 1993 and May 1995,84 patients with ischaemic heart disease with angiographically established coronary artery stenoses who had no history of stroke (ischaemic heart disease group; men:women=58:26, mean (SD) age $=61.1(9.6)$ years) and 200 age matched subjects with minor neurological disorders who had no history of ischaemic heart disease (control group; men:women $=117: 83$, mean $($ SD) age $=62.0(9.2)$ years) were screened with magnetic resonance angiography (MRA) for the presence of unruptured intracranial aneurysms. For all MRA positive patients, selective angiography was then undertaken.

Results-In the ischaemic heart disease group, the frequency of unruptured intracranial aneurysms established angiographically was $3.4 \%$ for men and $15.4 \%$ for women, compared with $2.6 \%$ and $3.6 \%$ respectively in the control group. Multiple logistic regression analyses disclosed that ischaemic heart disease was a significant and independent predictor for intracranial aneurysms in women.

Conclusions-These results suggest that in women ischaemic heart disease is a risk factor for intracranial aneurysms. Coexistence of intracranial aneurysms should be suspected in women patients with ischaemic heart disease.

(F Neurol Neurosurg Psychiatry 1998;64:536-538)
\end{abstract}

Keywords: coronary artery disease; cerebral aneurysm; angiography; magnetic resonance angiography; epidemiology
Although the pathogenesis of intracranial aneurysms still remains unclear, it has been pointed out that three main factorsdevelopmental faults in the media, atherosclerosis, and hypertension - have roles of varying importance according to the age at which the intracranial aneurysm develops. ${ }^{1}$ Atherosclerosis is often evident in patients with intracranial aneurysms. ${ }^{2}$ Postmortem studies documented the concurrence of coronary atherosclerotic lesions and intracranial aneurysms, suggesting that patients with intracranial aneurysms had three times the risk of coronary artery disease. ${ }^{13}$ Iwata et $a l^{4}$ reported that unruptured intracranial aneurysms were detected on intracranial intra-arterial digital subtraction angiography in four $(5.6 \%)$ of 72 consecutive patients who underwent coronary angiography for the diagnosis of angina pectoris. However, no systematic clinical study has been carried out and it remains uncertain whether ischaemic heart disease is a risk factor for intracranial aneurysms. Current advances in magnetic resonance angiography (MRA) allow this technique to be used in the detection of unruptured intracranial aneurysms ${ }^{5}$ instead of invasive selective angiography, currently the gold standard in the diagnosis of intracranial aneurysms. ${ }^{6}$ Examination by MRA, which has an acceptable sensitivity and specificity for detecting unruptured intracranial aneurysms, is suitable for a screening test. ${ }^{56}$ We studied the relation of ischaemic heart disease with intracranial aneurysms, comparing the prevalence of unruptured intracranial aneurysms in patients with ischaemic heart disease with that in age matched subjects with or without vascular risk factors who had no history of ischaemic heart disease. In this study, cerebral MRA was used for a screening measure for unruptured intracranial aneurysms and selective cerebral angiography was then used for verification in all MRA positive patients.

\section{Patients and methods}

There were patients with ischaemic heart disease and angiographically established coronary artery stenoses who had no history of stroke (ischaemic heart disease group) and 200 age matched patients with minor neurological disorders and various vascular risk factors who 
Table 1 Possible risk factors

\begin{tabular}{|c|c|c|c|c|c|c|}
\hline & \multicolumn{3}{|l|}{ Male } & \multicolumn{3}{|l|}{ Female } \\
\hline & $I H D(n=58)$ & Control $(n=117)$ & $p$ Value $^{*}$ & $I H D(n=26)$ & Control $(n=83)$ & $p$ Value $^{\star}$ \\
\hline Age $(y$, mean $(S D)$ & $59.0(9.1)$ & $59.8(9.1)$ & NSt & $65.7(9.3)$ & $65.0(8.5)$ & NSt \\
\hline Hypertension (n (\%)) & $17(29.3)$ & $23(19.7)$ & NS $\ddagger$ & $9(34.6)$ & $33(39.8)$ & NS $\ddagger$ \\
\hline Diabetes mellitus ( $\mathrm{n}(\%)$ ) & $18(31.0)$ & $10(8.5)$ & $<0.001 \ddagger$ & $6(23.1)$ & $12(14.5)$ & NS $\ddagger$ \\
\hline Hyperlipidaemia (n (\%)) & $24(41.4)$ & $14(12.0)$ & $<0.001 \ddagger$ & $9(34.6)$ & $25(30.1)$ & NS $\ddagger$ \\
\hline Smoking habit (n (\%)) & $36(62.1)$ & $70(59.8)$ & NS $\ddagger$ & $2(7.7)$ & $8(9.6)$ & NS $\ddagger$ \\
\hline
\end{tabular}

* Difference between the IHD and control groups.

† Student's $t$ test.

$\ddagger \chi^{2}$ test.

$\mathrm{IHD}=$ ischaemic heart disease; $\mathrm{NS}=$ not significant.

had no history of ischaemic heart disease (control group). The ischaemic heart disease group comprised 26 women and 58 men ranged in age from 40 to 79 (mean (SD) 61.1 (9.6)) years, and the control group comprised 83 women and 117 men ranged from 41 to 79 (62.0 (9.2)) years. The ischaemic heart disease group consisted of patients consecutively admitted to the cardiology service of our hospital for known ischaemic heart disease between October 1993 and May 1995 whose coronary angiography showed coronary artery stenoses and who agreed to enter the study. Critically ill patients, those with a history of stroke, and those who had a pacemaker were excluded. The control population was selected from a list of patients with minor neurological disorders seen in the same period who agreed to take part in the study, detailing age and sex only. Patients with known or suspected ischaemic heart disease, and those with intracranial haemorrhages, intracranial neoplasms, or head traumas were excluded. Sixty patients had a single lacunar stroke, and 140 patients had other non-specific neurological complaintsfor example, muscle contraction headache, migraine, and dizzy spells. Informed consent was obtained from all subjects before they entered the study. Unruptured intracranial aneurysms were first screened with intracranial MRA, and selective cerebral angiography was then undertaken in all MRA positive patients for verification. All MRA examinations were performed with a 1.0 tesla system (Magnetom Impact; Siemens) and a validated procedure for evaluation of intracranial arteries. ${ }^{7}$ Images were acquired by a three dimensional, Fourier transformed time of flight method. The indices of intracranial MRA were repetition time of $35-40 \mathrm{~ms}$, echo time of $7 \mathrm{~ms}$, and a flip angle of $15^{\circ}$. The number of excitations was one. We used two volume slab orientations, a horizontal section, and a section parallel to the clivus, thereby covering the view and compensating the signal attenuation due to flow direction. A $128 \times 128 \times 64$ matrix was used, with a field of view of $120 \times 120 \mathrm{~mm}$ and a partition thickness

Table 2 Correlations between risk factors and intracranial aneurysms by multiple logistic regression analysis in women

\begin{tabular}{llll}
\hline Variable & Odds ratio & $95 \%$ CI & p Value \\
\hline Ischaemic heart disease & 7.025 & $1.239-39.824$ & 0.028 \\
Age (by 1 year) & 0.971 & $0.885-1.064$ & 0.526 \\
Hypertension & 2.463 & $0.424-14.323$ & 0.316 \\
Diabetes mellitus & 0.447 & $0.041-4.870$ & 0.509 \\
Hyperlipidaemia & 1.391 & $0.257-7.525$ & 0.702 \\
Smoking habit & 4.708 & $0.619-35.824$ & 0.135 \\
\hline
\end{tabular}

of $1 \mathrm{~mm}$, resulting in a $0.94 \times 0.94 \times 1 \mathrm{~mm}$ voxel. The axial images thus acquired were used as source data for the construction of projection images with the use of a maximum intensity pixel projection algorithm. In each case, nine projection images at $22.5^{\circ}$ increments vertical and horizontal over a $180^{\circ}$ range were provided. Two investigators (TU, MT) who were blinded to all clinical information independently reviewed all MRAs for the presence of intracranial aneurysms. For all patients whose MRA findings were rated as positive by either of the reviewers, selective angiography was then undertaken. For risk factor evaluation, we recorded the presence or absence of hypertension, diabetes mellitus, hyperlipidaemia, and smoking habit in all subjects. All female patients were also checked for use of oral contraceptives. Hypertension was judged as present when repetitive measurements of blood pressure showed a systolic pressure above $160 \mathrm{~mm} \mathrm{Hg}$ or a diastolic pressure above $95 \mathrm{~mm} \mathrm{Hg}$, or when the patient had a history of treatment for hypertension. Diabetes mellitus was diagnosed when a fasting blood glucose concentration was above $140 \mathrm{mg} / \mathrm{dl}$ or when the patients had a history of treatment for diabetes mellitus. Hyperlipidaemia was defined when a serum lipid examination showed a serum total cholesterol concentration above $220 \mathrm{mg} / \mathrm{dl}$, triglyceride concentration above $150 \mathrm{mg} / \mathrm{dl}$, or HDL cholesterol concentration below $40 \mathrm{mg} / \mathrm{dl}$, or when the patients had a history of treatment. As a sex related difference in the frequency of intracranial aneurysms has been well recognised, ${ }^{9-12}$ the analysis was performed for each sex. Multiple logistic regression analysis was used to estimate independent effects of the predictive variables on intracranial aneurysms. All statistical analyses were carried out with SAS Release 6.10 software. The level of significance was set at $\mathrm{p}<0.05$ for all analyses.

\section{Results}

Of 17 MRA positive patients, intracranial aneurysms were verified on selective angiography in 12 , including seven aneurysms located on the middle cerebral artery (MCA), four on the internal carotid-posterior communicating artery (IC-PCoA), two on the vertebralposterior inferior cerebellar artery, and one on the cavernous portion of the internal cerebral artery (ICA). One patient in the ischaemic heart disease group had three aneurysms (one on the MCA, one on the IC-PCoA, and one on 
the cavernous portion of the ICA). The diameter of the aneurysms varied from $3 \mathrm{~mm}$ to 7 $\mathrm{mm}$. The causes of the five MRA false positives (on the anterior communicating arteries in two patients, on the IC-PCoA in two patients, and on the MCA trifurcation in one patient) became subsequently evident on selective angiography, including formation of loops, overlapping of vessels, and IC-PCoA infundibular dilatation. In the patient with multiple aneurysms, an aneurysm on the ICA had been overlooked on MRA. Intracranial aneurysms were found in six $(7.1 \%)$ of 84 patients in the ischaemic heart disease group and six $(3.0 \%)$ of 200 patients in the control group. The frequencies were $15.4 \%$ (four of 26) for women and $3.4 \%$ (two of 58 ) for men in the ischaemic heart disease group, and $3.6 \%$ (three of 83 ) for women and $2.6 \%$ (three of 117) for men in the control group. Table 1 summarises the possible risk factors. In women, there was no significant difference between the ischaemic heart disease and control groups, whereas in men the incidence of diabetes mellitus and hyperlipidaemia was significantly higher in the ischaemic heart disease group than in the control group. No female patients in either group had used oral contraceptives. Multiple logistic regression analyses disclosed that ischaemic heart disease was a significant and independent predictor for intracranial aneurysms in women (table 2) but not in men, in whom there were only a few intracranial aneurysm positive patients.

\section{Discussion}

According to several large postmortem series, the prevalence of incidental intracranial aneurysms is $2 \%$ to $5 \% .^{9}$ In recent angiographic studies, the frequency of asymptomatic aneurysms ranged from $1 \%$ to $6.5 \% .{ }^{10}{ }^{13}$ Although the prevalence of unruptured intracranial aneurysms may vary with constituents of the population-for example, age, sex, race, and risk factors - and the sensitivity may vary with each method of examination, our results, except for those in female patients with ischaemic heart disease, were comparable with those of previous studies. The incidence of intracranial aneurysms was predominant in women and increased with age. ${ }^{9-12}$ Several cohort studies have reported that hypertension ${ }^{9111215}$ and smoking $^{15-17}$ predisposed to subarachnoid haemorrhage. A systematic review showed that smoking, hypertension, and alcohol misuse were important risk factors for subarachnoid haemorrhage. ${ }^{18}$ Oral contraceptives was also considered as a risk factor. ${ }^{17}{ }^{19}$ In this study multiple logistic regression analyses, in which age, hypertension, and smoking habit were considered as independent variables, showed that only ischaemic heart disease was a significant and independent predictor for intracranial aneurysms in women. Previous studies ${ }^{911}$ showed that there was a male predominance in the incidence of intracranial aneurysms under 20 years of age but a female predominance in patients over 60 years of age. The effect of recurrent pregnancies on the connective tissue and rapid progress of atherosclerotic change after the menopause could play some part in aneurysmal formation in women. ${ }^{9}{ }^{10} 12 \mathrm{~A}$ population based case-control study found postmenopausal women to be at higher risk for subarachnoid haemorrhage than premenopau-

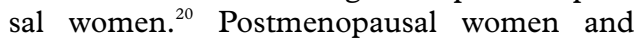
women who have undergone oophorectomy reportedly also had a higher risk of coronary artery disease than premenopausal women of similar ages. ${ }^{21}$ Thus for postmenopausal women a common mechanism such as decreased oestrogen concentrations could affect formation of intracranial aneurysms and coronary atherosclerosis.

In conclusion, our results suggest that the coexistence of intracranial aneurysms is highly suspected in female patients with ischaemic heart disease. Our finding is to be further substantiated in a larger population based study.

This work was supported by a research grant for cardiovascular diseases $(8 \mathrm{C}-4)$ from the Ministry of Health and Welfare, Japan.

1 Crawford T. Some observations on the pathogenesis and natural history of intracranial aneurysms. $\mathcal{f}$ Neurol Neurosurg Psychiatry 1959;22:259-66.

2 Adamson J, Humphries SE, Ostergaard JR, et al. Are cerebral aneurysms atherosclerotic? Stroke 1994;25:963-6.

Sarner M, Crawford MD. Ruptured intracranial aneurysm: clinical series. Lancet 1965;ii:1251-4.

4 Iwata K, Misu N, Terada K, et al. Screening for unruptured asymptomatic intracranial aneurysms in patients undergoasymptomatic intracranial aneurysms in patients under

ing coronary angiography. $\mathcal{F}$ Neurosurg $1991 ; 75: 52-5$.
5 Korogi $\mathrm{Y}$, Takahashi $\mathrm{M}$, Mabuchi N, et al. Intracranial aneurysms: diagnostic accuracy of three-dimensional, Fourier transform, time-of-flight MR angiography. Radiology
nationrier transform, tim

6 Ross JS, Masaryk TJ, Modic MT, et al. Intracranial aneurysms: evaluation by MR angiography. AfNR 1990; 11:449-56.

7 Uehara T, Mori E, Tabuchi M, et al. Detection of occlusive lesions in intracranial arteries by three-dimensional timeof-flight magnetic resonance angiography. Cerebrovasc Dis 1994;4:365-70

8 Kawaguchi T, Fujita S, Hosoda K, et al. Diagnostic accuracy of the magnetic resonance angiography for unruptured cerebral aneurysms. Neuroradiology 1994;36:562-3.

9 Sekhar LN, Heros RC. Origin, growth, and rupture of saccular aneurysms: a review. Neurosurgery 1981;8:248-60.

10 Ujiie H, Sato K, Onda H, et al. Clinical analysis of incidentally discovered unruptured aneurysms. Stroke 1993;24: tally disco $1850-6$.

11 Andrews RJ, Spiegel PK. Intracranial aneurysms: age, sex, blood pressure, and multiplicity in an unselected series of blood pressure, and multiplicity in an
patients. $\mathscr{F}$ Neurosurg 1979;51:27-32.

12 Østergaard JR, Høg E. Incidence of multiple intracranial aneurysms: influence of arterial hypertension and gender. $\mathcal{f}$ Neurosurg 1985;63:49-55.

13 Atkinson JLD, Sundt TM Jr, Houser OW, et al. Angiographic frequency of anterior circulation intracranial aneurysms. F Neurosurg 1989;70:551-5.

14 Nakagawa $T$, Hashi $K$. The incidence and treatment of asymptomatic, unruptured cerebral aneurysms. $\mathcal{F}$ Neurosurg 1994;80:217-23.

15 Sacco RL, Wolf PA, Bharucha NE, et al. Subarachnoid and intracerebral hemorrhage: natural history, prognosis, and precursive factors in the Framingham study. Neurology 1984;34:847-54.

16 Longstreth WT Jr, Nelson LM, Koepsell TD, et al. Cigarette smoking, alcohol use, and subarachnoid hemorrhage. stroking, alcohol use,

17 Petitti DB, Wingerd J. Use of oral contraceptives, cigarette smoking, and risk of subarachnoid haemorrhage. Lancet 1978;ii:234-6

18 Teunissen LL, Rinkel GJE, Algra A, et al. Risk factors for subarachnoid hemorrhage: a systematic review. Stroke 1996;27:544-9.

19 Layde PM, Berai V, Kay CR. Further analyses of mortality in oral contraceptive users. Royal College of General Practitioners' oral contraception study. Lancet 1981;i:541-46.

20 Longstreth WT Jr, Nelson LM, Koepsell TD, et al. Subarachnoid hemorrhage and hormonal factors in women: a population-based case-control study. Ann Intern Med 1994;121:168-73.

21 Matthews KA, Meilahn E, Kuller LH, et al. Menopause and risk factors for coronary heart disease. N Engl F Med 1989; 321:641-6. 